\title{
Terapia Preventiva Pós Traumatismo Dental na Primeira Infância por Reabilitação Protética Funcional: Relato de Caso
}

\author{
Preventive therapy after dental trauma in early childhood by functional prosthetic \\ rehabilitation: Case report
}
Terapia preventiva post traumatismo dental en la primera infancia por rehabilitación protética funcional: Relato de caso

\begin{abstract}
Sâmia Caldas da Costa ${ }^{1 *}$, Thomas Anderson Santos do Amarall ${ }^{1}$, Dóris Kós Miranda ${ }^{1}$, Jorge Sá Elias Nogueira ${ }^{1}$, Yasmin Caldas Sanches dos Santos ${ }^{1}$, Amanda Souza Nunes ${ }^{1}$, Amujacy Tavares Vilhena ${ }^{1}$, Lucas Dias Silva ${ }^{1}$, Stephanny Amorim de Souza ${ }^{1}$, Raul Henriques dos Santos ${ }^{1}$, Julya Késsia Cruz Freitas ${ }^{1}$, Mayara Cristina Souza de Oliveira1.
\end{abstract}

\section{RESUMO}

Objetivo: Relatar um caso de traumatismo dental na primeira infância seguido de reabilitação protética funcional, dando um enfoque à utilidade dessa terapia nos pacientes infantis acometidos por tal situação. Detalhamento do Caso: Paciente do sexo feminino, 5 anos, compareceu à Clínica da Unidade Odontológica Infantil (UOI) do Centro Universitário do Estado do Pará (CESUPA) acompanhada de sua mãe, que historiou o acontecimento de trauma dental aos 2 anos de vida da sua filha por motivo de queda em um momento de lazer com velocípede. Levando em conta a gravidade do trauma e a preocupação na preservação do espaço até a erupção do germe do dente permanente, indicou-se a confecção e instalação de um aparelho protético funcional removível com dente de estoque. No controle, observou-se boa adaptação do aparelho pela criança e funcionalidade na fonética, estética e manutenção de espaço para o dente permanente, considerando-se a eficácia da terapia para prevenção de hábitos nocivos e maloclusões futuras. Discussão: As quedas infantis são descritas na literatura como a maior causa de traumas dentais, situação que foi identificada no referido caso. Para uma eficaz reabilitação de traumatismo dental, o tratamento deve ser imediato, obedecendo ao tipo de envolvimento causado às estruturas peri-dentais.

Palavras-chave: Traumatismo, Reabilitação bucal, Odontopediatria.

\begin{abstract}
Objective: To report a case of dental trauma in early childhood followed by functional prosthetic rehabilitation, focusing on the usefulness of this therapy in the infantile patients affected by this situation. Case Report: Female patient, 5 years old, attended the Clinic of the Infant Dental Unit (UOI) of the University Center of the State of Pará (CESUPA) accompanied by her mother, who reported the dental trauma event at the 2 years of her daughter's life for reasons of falling into a moment of leisure with a velocipede. Taking into account the severity of the trauma and the preoccupation in the preservation of the space until the eruption of the permanent tooth germ, it was indicated the confection and installation of a functional prosthetic device removable with tooth of stock. In the control, it was observed a good adaptation of the device by the child and functionality in phonetics, aesthetics and maintenance of space for the permanent tooth, considering the effectiveness of the therapy to prevent harmful habits and future malocclusions. Discussion: Infant falls are described in the literature as the major cause of dental trauma, a situation that was identified in the aforementioned case. For effective rehabilitation of dental trauma, the treatment should be immediate, obeying the type of involvement caused by peri-dental structures.
\end{abstract}

Key words: Trauma, Oral rehabilitation, Pediatric dentistry.

${ }^{1}$ Centro Universitário do Estado do Pará, Belém-PA. *E-mail: samiacaldas@gmail.com 


\section{RESUMEN}

Objetivo: describir un caso de traumatismo dental en la primera infancia seguido de rehabilitación protética funcional, dando un enfoque a la utilidad de esa terapia en los pacientes infantiles acometidos por tal situación. Detalle del Caso: El paciente de sexo femenino, de 5 años, assistido em la Clínica de la Unidad Odontológica Infantil (UOI) del Centro Universitario del Estado de Pará (CESUPA) acompañada de su madre, que historió el acontecimiento de trauma dental en los 2 años de vida de su hija por motivo de caída en un momento de ocio con velocípedo. Teniendo en cuenta la gravedad del trauma y la preocupación en la preservación del espacio hasta la erupción del germen del diente permanente, se indicó la confección e instalación de un aparato protético funcional removible con diente de stock. En el control se observó buena adaptación del aparato por el niño y funcionalidad en la fonética, estética y mantenimiento de espacio para el diente permanente, considerando la eficacia de la terapia para prevención de hábitos nocivos y maloclusiones futuras. Discusión: Las caídas infantiles se describen en la literatura como la mayor causa de traumas dentales, situación que fue identificada en el referido caso. Para una eficaz rehabilitación de traumatismo dental, el tratamiento debe ser inmediato, obedeciendo al tipo de envoltura causado a las estructuras peridentales.

Palabras clave: Traumatismo, Rehabilitación bucal, Odontopediatría.

\section{INTRODUÇÃO}

O trauma dental pode ser conceituado como uma agressão térmica, química ou mecânica sofrida pelo dente e estruturas adjacentes, cuja potência supera a resistência encontrada nos tecidos ósseo e dentário. Sua extensão possui relação direta com a intensidade, tipo e duração do impacto (BIJELLA MFT, 1990; DUARTE DA, 2001).

Com os baixos índices atuais de cárie dentária verificadas no Brasil, outros agravos de saúde bucal vêm despertando o interesse da comunidade científica, e o traumatismo dentário (TD) está entre as patologias. Em relação a etiologia, os altos índices de violência, acidentes de trânsito e uma maior participação das crianças em atividades esportivas contribuem para transformar o TD em um problema crescente de saúde pública, principalmente em Odontopediatria, pela razão de trazer muitos gastos para a saúde pública e privada e ainda existir a grande dificuldade pertencente ao seu tratamento (MARCENES W, et al., 1999; BROWN CJ, 2002). Na primeira infância, os traumatismos dentários em dentes decíduos são acidentes comuns, onde podem causar tanto danos estéticos como funcionais, prejudicando assim a qualidade de vida da criança (VASCONCELLOS RRDH, et al., 2003; GRANVILLE LM, et al., 2009).

Existe um predomínio de traumas bucais na primeira infância, que é justamente quando a criança aprende a se levantar, andar e correr. Nesta fase específica, a criança possui falta de coordenação motora em virtude de sua pouca idade. Em relação ao tipo de trauma na dentição decídua, o mais corriqueiro é a queda da criança de sua própria altura, chegando a $80 \%$ dos casos. Das crianças entre 1 a 3 anos, sem discrepância entre meninos e meninas, aproximadamente 11 a $30 \%$ possuem algum trauma dental (LOSSO EM et al., 2011). O atendimento a crianças com traumatismo dental na dentição decídua requer uma abordagem diferenciada, por conta da intima relação entre o dente decíduo afetado pelo trauma e o germe do dente permanente sucessor (LOSSO EM et al., 2011).

Os dentes decíduos são fundamentais no crescimento e desenvolvimento da criança e contribuem nas funções mastigatórias, articulatórias, fonéticas, estéticas e também oclusais, sendo classificados como verdadeiros mantenedores de espaço naturais. Entretanto, a perda precoce dos mesmos é uma situação que pode ocorrer, principalmente nos episódios de cárie e traumatismo dentário (GONÇALVES LM, et al., 2013; BONECKER M, et al., 2011; CORREA MSNP et al., 2011; GUEDES AC, et al., 2012; SOUSA JM, et al., 2012).

Os mantenedores de espaço artificiais, por meio da reposição protética dos elementos dentais, têm por objetivo a devolução da integridade das arcadas, promovendo o restabelecimento das funções normais como mastigação, deglutição e fonação para evitar a instalação de hábitos nocivos e maloclusões (BESSADET M, 
et al., 2013; PEREIRA L, et al., 2010). A finalidade deste tratamento é de prevenir males futuros, além de proporcionar uma melhora na saúde geral do paciente, aumentando assim sua autoestima e convivência social, onde interfere de forma positiva na qualidade de vida da criança e familiares (MISHRA A, et al., 2013).

Haja vista a complexidade da abordagem do paciente infantil com histórico de traumatismo dentário, o presente artigo tem como objetivo relatar um caso de traumatismo dental na primeira infância seguido de reabilitação protética funcional, dando um enfoque a utilidade dessa terapia nos pacientes infantis acometidos por tal situação.

\section{DETALHAMENTO DO CASO}

Paciente do sexo feminino, 5 anos de idade, compareceu à Clínica da Unidade Odontológica Infantil (UOI) do Centro Universitário do Estado do Pará (CESUPA) acompanhada de sua mãe, que historiou o acontecimento de trauma dental aos 2 anos de vida da sua filha por motivo de queda em um momento de lazer com velocípede. Foi realizado o exame clínico e radiográfico da região afetada pelo trauma, observandose a ausência do elemento 51 (incisivo central superior direito) (Figura 1 e 2), mas com a presença de um pequeno fragmento deste na gengiva e a fratura incisal do elemento 61 (incisivo central superior esquerdo).

Figura 1 - Paciente com perda traumática do elemento 51 e com o elemento 61 devidamente restaurado.

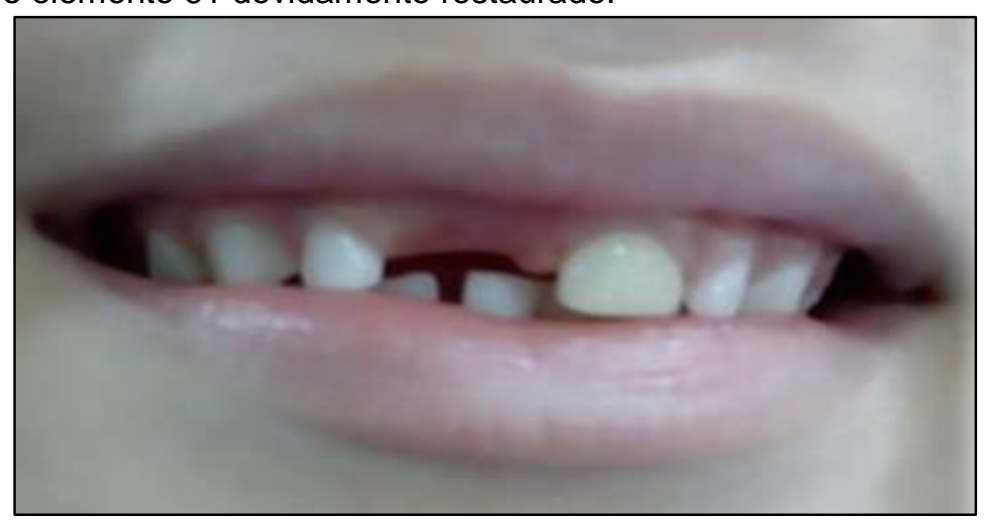

Fonte: Autoria própria, 2018.

Levando em consideração a gravidade do trauma e a preocupação em relação a preservação do espaço até a erupção do germe do dente permanente, no plano de tratamento da criança foi sugerido à responsável, a confecção de um aparelho protético funcional removível com dente de estoque.

Figura 2 - Ausência do elemento 51 em radiografia periapical.

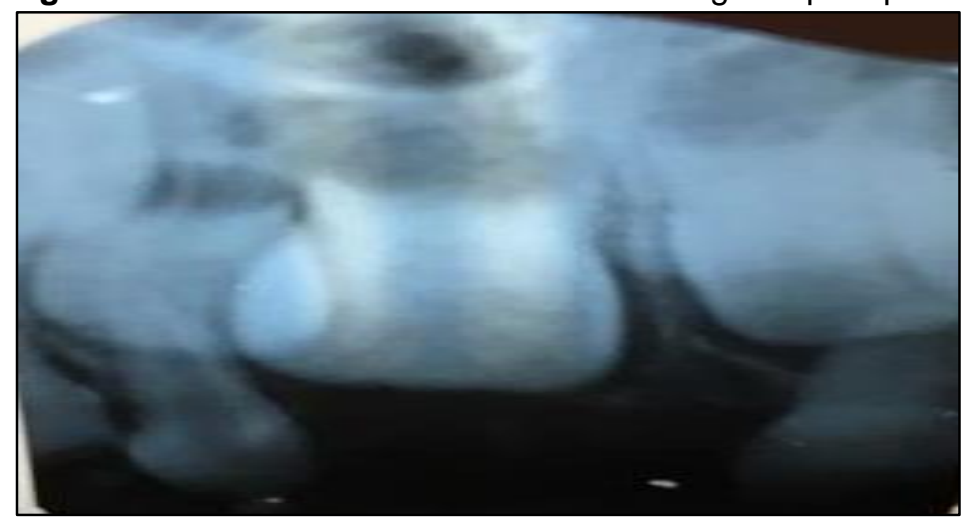

Fonte: Autoria própria, 2018. 
Mediante o termo de consentimento da mãe para a execução do tratamento, a consulta seguinte consistiu na restauração do elemento 61 com resina composta na cor B2 (Figura 1), seguida da cirurgia de remoção do fragmento do elemento 51 que foi avulsionado pelo trauma. Em uma nova sessão, foi realizada a moldagem das arcadas superior e inferior obtendo-se os modelos de trabalho para posterior construção do aparelho protético superior em resina acrílica (Figura 3).

Figura 3 - Modelos de trabalho, superior e inferior.

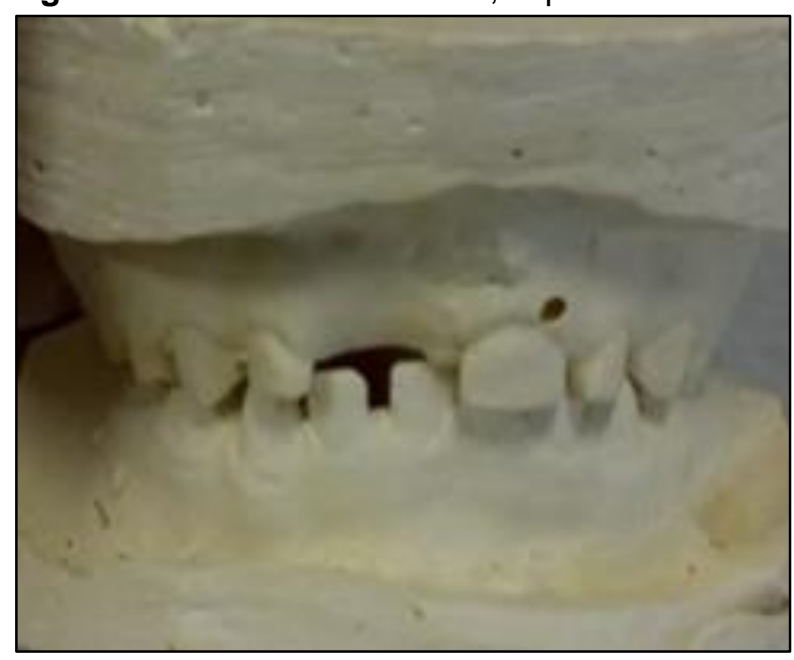

Fonte: Autoria própria, 2018.

No aparelho, foram confeccionados grampos de retenção extendidos na região vestibular e foi adaptado um dente de estoque decíduo (51) em resina acrílica preenchendo-se o espaço referente à perda (Figura 4).

Figura 4 - Aparelho em acrílico com dente de estoque e grampos de extensão vestibular.

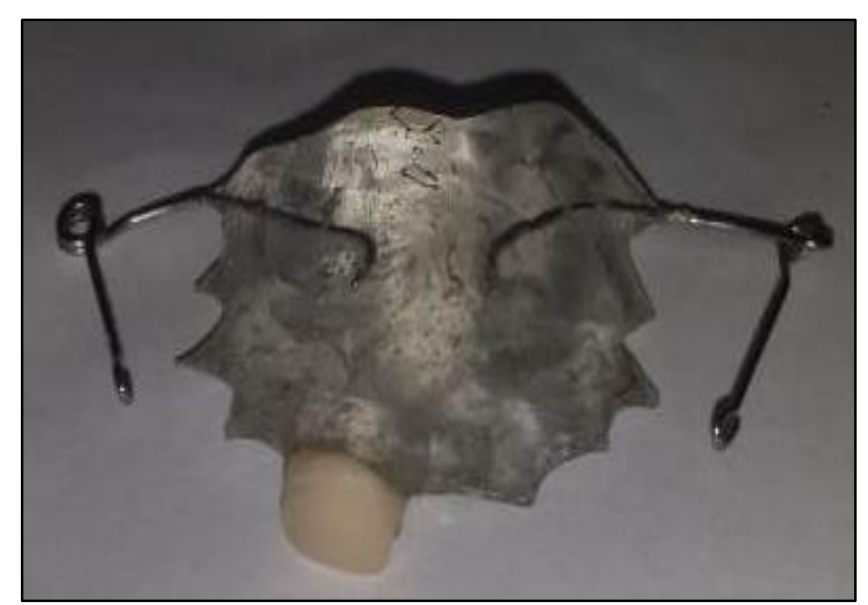

Fonte: Autoria própria, 2018.

O aparelho acabado e polido foi instalado na paciente infantil (Figura 5) e para aumentar sua estabilidade, realizou-se o acréscimo de pequena porção de resina composta ("stops") nas superfícies vestibulares dos dentes 55, 54, 65 e 64 de modo que, os grampos de extensão vestibular não se deslocassem em sentido oclusal. Recomendações com relação ao uso do aparelho foram feitas à sua responsável e também a criança, em abordagem apropriada a sua idade. 
Figura 5 - Paciente reabilitada com prótese removível confeccionada com dente de estoque.

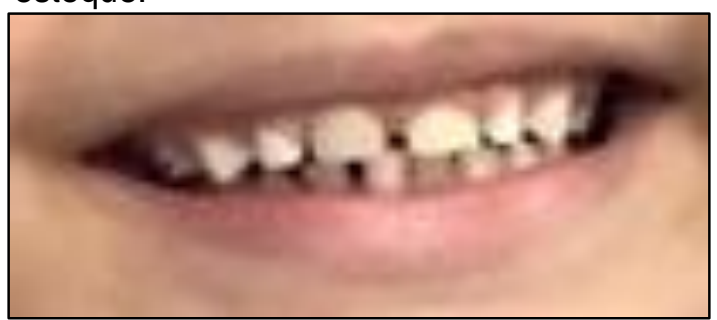

Fonte: Autoria própria, 2018.

A paciente retornou para as consultas de controle com o relato de boa adaptação ao aparelho e de satisfatória recuperação das funções de fonética, estética e manutenção de espaço para o dente permanente. Foi alertado a responsável à necessidade de proservação clínica e radiográfica para definição do momento adequado de retirada definitiva do aparelho.

\section{DISCUSSÃO}

Não são raros os traumatismos dentais na dentição temporária, visto que eles possuem uma prevalência agregada de $26 \%$ no Brasil e de $23 \%$ no mundo, sendo essas porcentagens propensas de aumento com 0 passar dos anos (ANDREASEN JO et al., 1994; ALDRIGUI JM, 2012).

Diante do caso clinico exposto, a responsável pela paciente relatou um trauma decíduo na região anterior devido a um momento de lazer da criança em um brinquedo de andar, razão esta compatível com os relatos de que os acidentes na primeira infância geralmente acontecem quando a criança está desacompanhada ou principalmente dentro da sua própria casa (FLORES MT et al., 2002).

O acidente aconteceu quando a criança possuía apenas 2 anos de idade, sendo essa faixa etária a de maior incidência de traumatismo dentoalveolar, pois nesse período a criança ainda não possui coordenação motora para evitar quedas e promover autoproteção, justificando a ocorrência deste tipo de lesão (ASSUNÇÃO LRS et al., 2007; VASCONCELLOS RRDH et al., 2003).

As quedas infantis são descritas na literatura como a maior causa de traumas dentais, situação essa que foi identificada no referido caso. Também foi identificado o momento tardio em que foi buscada a atenção odontológica. Casos assim são frequentes, tornando-se preocupante saber que nem sempre os pais procuram esse atendimento, mesmo quando sabem que o filho sofreu traumatismo. Em um estudo realizado por Bonini CVAG (2009), foi encontrado que mais de $90 \%$ dos casos de TD na fase decídua não são tratados e que somente uma pequena parte dos pais procura atendimento nas primeiras 24 horas (BONINI CVAG et al., 2009).

Um ano depois, Viegas CM (2010) fez um novo levantamento com 112 pais, e os mesmos relataram que o filho tinha histórico de traumatismo, porém menos da metade procurou o cirurgião dentista, mostrando que, por diversas vezes, os pais esquecem por um tempo que a criança sofreu algum tipo de traumatismo, principalmente se forem mais leves. Em geral, os pais só se atentam a casos mais graves, que necessitam de tratamento odontológico imediato (VIEGAS CM et al., 2010).

Diversos autores discorreram sobre as variadas causas de TD, que incluem quedas de superfícies elevadas ou da própria altura, quedas de bicicleta, acidentes esportivos e automobilísticos, além de outras, como lesões iatrogênicas nos recém-nascidos, doenças convulsivas, agressões físicas e maus tratos (NICOLAU B et al., 2001; ALEXANDRE GC et al., 2000; OLIVEIRA BH et al., 1995).

É mensurável o impacto negativo que o trauma dental exerce na qualidade de vida de uma criança, e isto está atrelado diretamente a sua gravidade e suas sequelas na dentição decídua (ALDRIGUI JM, 2011). Sequelas que tanto podem atingir estruturas periodontais, ósseas e de tecido mole, variando de acordo com 
a intensidade e o tipo de trauma ocorrido. Fraturas, luxações e avulsões são as possíveis consequências para esta dentição temporária (WANDERLEY MT et al., 2014, ROCHA VG et al., 2008). O caso da paciente em questão teve a consequência mais severa de TD, que foi a avulsão, onde ocorreu o deslocamento completo do dente para fora de seu alvéolo (LOSSO EM et al., 2011). Nestes casos, é necessária uma abordagem de tratamento mais específica para que se atinja o sucesso terapêutico e se dê a garantia da menor possibilidade de lesão do germe do dente permanente.

De acordo com a literatura, o incisivo central superior, que foi o elemento atingido pelo trauma da paciente em pauta, é o mais envolvido nesses casos. A faixa etária de crianças atingidas varia de 1 a 4 anos (ROCHA VG et al., 2008). Nos casos de avulsão dentária, é pacto entre todos os autores de que não se reimplante o dente decíduo, pois isso pode gerar prejuízos para o desenvolvimento do germe permanente sucessor. Quando esses dentes são reimplantados causam uma série de complicações potenciais, tais como reabsorção inflamatória, anquilose, infecção e esfoliação, além de danos ao dente permanente, como hipoplasia de esmalte, dilaceração coronária ou radicular e sequestro do germe permanente (GRANVILLE G et al., 2009; GONDIN OJ et al., 2011).

A abordagem de relevância para a dentadura decídua é bastante pertinente, tanto para os pais quanto para os profissionais de saúde. A crença de que o "dente de leite" será substituído por outro dente "sadio" levam os pais a negligenciarem o tratamento do trauma na dentição decídua. Isto acontece pela falta de informações sobre a necessidade e importância do tratamento (MAIA LC et al., 2012), o que foi intensamente abordado com a responsável da paciente do caso, para que a mesma pudesse entender e concordar com a reabilitação proposta.

Não é pelo motivo desses elementos se manterem na cavidade oral por um período temporário, que não se deve ter a preocupação de sua perda precoce, seja por cárie ou por esta situação de traumatismo dentário. Os dentes decíduos são fundamentais no crescimento e desenvolvimento da criança e são responsáveis pela boa evolução do sistema estomatognático (BIJOOR RR et al., 2005).

Por essa razão, imediatamente foram refletidas as consequências futuras as quais a paciente infantil poderia estar sujeita, com a avulsão do elemento dental pelo traumatismo. A literatura evidencia que a perda precoce antes da esfoliação natural de um dente decíduo pode gerar verdadeiros descompassos na dentição permanente, como alterações ortodônticas (pela inclinação dos dentes vizinhos, interferência na função mastigatória, perda de espaço, extrusão do antagonista, deglutição atípica), alterações fonéticas (na articulação das palavras) e alterações estéticas (pela ausência do elemento dentário). Tudo isto aliado a possível perda de espaço para a erupção do dente permanente, propiciando a diminuição de comprimento no arco dentário e ainda podendo criar hábitos deletérios devido a alteração maxilar que causa uma postura incorreta da língua (COELHO MLG, 2003; NOBREGA ML et al., 2018).

Pensando nesse âmbito da prevenção e na reabilitação estética-funcional da criança acometida pelo trauma, foi conveniente a sugestão da confecção do aparelho funcional para a paciente, agindo-se de forma interceptiva a todas as alterações citadas e oportunizando-se o desenvolvimento da dentição permanente de maneira satisfatória. Está bem comprovada a eficácia de próteses funcionais no tratamento de crianças acometidas pelo trauma dental, pois também se acredita que a perda precoce dos elementos dentais anteriores influencia no comportamento social de crianças e adolescentes, levando a problemas psicológicos e prejuízo a nível emocional da criança (SILVA MCVS et al., 2011, NOBREGA ML et al., 2018).

O aparelho proposto à paciente apresenta a função de mantenedor de espaço estético-funcional, e poderia ser tanto fixo por aço inoxidável ou removível de resina acrílica, tendo sua indicação na dependência do caso. Neste caso clínico, foi escolhida a confecção do mantenedor de espaço removível em acrílico, mencionandose que, certos autores, discordam da utilização desse tipo em crianças menores de cinco anos, justamente pela falta de cooperação do paciente (COSTA ICA et al., 2015).

Ainda assim, este foi utilizado devido a paciente ter um certo grau de maturidade e colaboração, o que influenciou a escolha em caráter positivo, além do fato da avulsão traumática ter se restringido a um único elemento dentário. Também foi levada em consideração, a simplicidade de se construir o aparelho, dele ser 
de baixo custo e ter facilidade no seu ajuste. Com ênfase nas vantagens do aparelho removível com dente estão ainda a possibilidade de reabilitar estética e funcionalmente o segmento anterior, manter simultaneamente o espaço para a erupção do sucessor permanente, impedir a extrusão do dente antagonista e permitir sua remoção para a apropriada higiene bucal da criança. Entretanto, as desvantagens em relação ao uso podem existir e foram dialogadas com a responsável da paciente, como o risco de perda ou quebra do aparelho, além da necessidade de colaboração da criança quanto aos cuidados e uso contínuo (PEREIRA et al., 2010).

Para uma eficaz reabilitação de TD, o tratamento deve ser imediato, seguindo um determinado protocolo envolvendo considerações médicas, odontológicas e sociais, sendo reforçado que o tratamento deve obedecer ao tipo de envolvimento causado às estruturas dentais e peri-dentais, além de tentar reduzir suas sequelas (TRAEBERT J et. al., 2006; BASTONE EB et al., 2000).

A maneira mais eficiente de prevenir tais traumatismos, principalmente em atividades esportivas, é a utilização do protetor bucal, que é confeccionado em consultório odontológico (ANDRADE R et al., 2010; COLLARES $\mathrm{K}$ et al., 2013). Há autores que salientam a necessidade da conscientização do uso do protetor bucal, visando estimular os praticantes de esportes e atividades, a atuar de maneira adequada e segura (CORREA MB et al., 2012). Entretanto, ainda não há estudos que enfatizem uma prevenção similar a esta nos pacientes infantis que estão na idade mais predisposta a traumatismos dentais, como a primeira infância.

\section{REFERÊNCIAS}

1- ANDRADE R, et al. Prevalence of dental trauma in Pan American games athletes. Dental traumatology: official publication of International Association for Dental Traumatology, v. 26, n. 3, p. 248-53, 2010.

2- ANDREASEN JO et al. Textbook and Color Atlas of Traumatic Injuries to the Teeth. 3rd ed., Copenhagen: Mosby, 1994. p.15180 .

3- ALDRIGUI JM. Prevalência de traumatismo em dentes decíduos e fatores associados: revisão sistemática e meta-análise. Tese de Doutorado. Universidade de São Paulo, 2012.

4- ALEXANDRE GC et al. Luxação Intrusiva de Dentes Decíduos. Revista da Assoc Paul Cirur Dent, São Paulo, v. 54; n. 3, p. 215219, maio/jun. 2000.

5- $\quad$ ASSUNÇÃO LRS et al. Análise dos traumatismos e suas sequelas na dentição decídua: uma revisão da literatura. Pesq Bras Odontoped Clín Integr. 2007.

6- BASTONE EB, et al. Epidemiology of dental trauma: A review of the literature. Aust. Dent. J. 2000; 2:9-45(1).

7- BESSADET M, et al. Impact of removable partial denture prosthesis on chewing efficiency. J Appl Oral Sci.2013;21(5):392-6.

8- BIJELLA MFT, et al. Occurrence of primary incisor traumatism in brazilian children: A house-by-house survey. J Dent Child, Chicago, v. 21, n. 6, p. 424-427, Nov./Dec. 1990.

9- BIJOOR RR, KOHLI K. Contemporary maintenance for the pediatric. N Y State Dent J.71(2):32-5, 2005.

10- BÖNECKER M, et al. Estética em Odontopediatria: Considerações Clínicas. Santos: Santos, 2011. 206 p.

11- BONINI CVAG, et al. Trends in the prevalence of traumatic dental injuries in Brazilian preschool children. Dental Traumatology 2009; 25: 594-598.

12- BROWN CJ. The management of traumatically intruded permanent incisors in children. Dent Update, v.29, n.1, p.38-44, Jan. /Feb. 2002.

13- CARDOSO M, ROCHA MJC. Mantenedor de espaço estético: uma solução para dentes decíduos traumatizados. Rev Ibero-am Odontopediatr Odontol Bebê 2004 nov-dez;7(40):512-8.

14- COELHO MLG. Perda precoce da dentição decídua: análise da percepção das mães de crianças de 02 a 06 anos de idade na sede do distrito de Jaibaras, Sobral-CE. [Mono-grafia] Sobral: Universidade Estadual do Vale do Acaraú; 2003.

15- COLLARES $\mathrm{K}$, et al. Effect of wearing mouthguards on the physical performance of soccer and futsal players: a randomized crossover study. Dental traumatology: official publication of International Association for Dental Traumatology, n. 14, p. 1-5, 2013.

16- CORREA MB, et al. Survey on the occurrence of dental trauma and preventive strategies among Brazilian professional soccer players. Journal of applied oral science: revista FOB, v. 18, n. 6, p. 572-6, 2010.

17- CORREAA MSNP. Odontopediatria na primeira infância. 3. ed. Santos: Santos, 2011. 942 p.

18- CORREA, M. et al. Video analysis of craniofacial soccer incidents: a prospective study. Journal of Science and Medicine in Sport / Sports Medicine Australia, v. 15, n. 1, p. 14-8, 2012.

19- COSTA ICO, et al. Mantenedor de Espaço Estético-Funcional em Odontopediatria-Prótese Fixa de Denari Relato de Caso Clínico. Fundação Faculdade de Odontologia. 2015

20- DUARTE DA, et al. Caderno de odontopediatria. Lesões Traumáticas em Dentes Decíduos: Tratamento e Controle. São Paulo: Santos, $2001.45 \mathrm{p}$

21- FLORES MT. Traumatic injuries in the primary dentition. Dent Traumatol. 2002; 18:287-98. 
22- GONDIN OJ et al. Sequelas em dentes permanentes após trauma nos predecessores decíduos e sua implicação clínica. RGO Gaúcha Odontol. 2011; 59(0): 113-120.

23- GONÇALVES LM, et al. Uso de prótese fixa adesiva como mantenedor de espaço em dentes anteriores decíduos: um relato de caso. Archives of Oral Research, v. 9, n.1, p. 85-90, 2013.

24- GRANVILLE G, et al. Conhecimento do cirurgião-dentista sobre avulsão dental no Programa de Saúde da Família de Campina Grande, PB, Brasil. Revista Odonto 2009; 33(1): 35-41.

25- GUEDES-PINTO AC. Odontopediatria. 8. ed. Santos: Santos, 2012. 1064 p.

26- LOSSO EM et al. Traumatismo dentoalveolar na dentição decídua SBO. 2011 Jan-Mar;8(1):114:e1-20.

27- MAIA LC, PRIMO LG, IAZZETTI GJ. Odontologia integrada na infância. São Paulo: Santos, 2012. 270 p.

28- MARCENES W, et al. Epidemiology of traumatic dental injuries to permanent incisors of schoolchildren aged 9 to 12 in Damascus, Syria. Endod Dent Traumatol 1999; 15: 117 - 23.

29- MISHRA A, et al. A pedoprosthetic rehabilitation in patients with severe early childhood caries (S-ECC). BMJ Case Rep 2013; 9:13.

30- NICOLAU B et al. Prevalence, causes and correlates of traumatic dental injuries among 13-yearolds in Brazil. Dent Traumatol. 2001; 17:213-7.

31- NOBREGA ML, et al. Implicações da perda precoce em odontopediatria. Revista Pró-UniverSUS. 2018 jan.jun.; 09 (1): $61-67$.

32- OLIVEIRA BH et al. Intrusão de incisivos decíduos provocando distúrbio no desenvolvimento de dentes permanentes: relato de caso. Rev Bras Odontol. 1995; 52:42-45.

33- PEREIRA L, MIASATO JM. Mantenedor de espaço estético-funcional em Odontopediatria. Revista de Odontologia da Universidade Cidade de São Paulo, 22(2): 154-62, mai-ago, 2010.

34- ROCHA VG et al. Freqüência dos traumatismos na dentição decídua: Estudo longitudinal descritivo. Arq Bras Odontol, 4(1): 3-10, 2008.

35- SILVA MCVS, et al. Reabilitação Oclusal com Prótese Parcial Removível Provisória Tipo "Overlay": Relato de Caso. R Bras Cienc Saúde.2011;15(4):455-60.

36- SOUSA JM, et al. Utilização de prótese parcial fixa modificada na primeira infância: relato de caso. Odontologia Clínico-Científica (Online), v. 11, n. 3, p. 253-257, 2012.

37- TRAEBERT J, et al. A etiology and rates of treatment of traumatic dental injuries among 12-year-old school children in a town in southern Brazil. Dental Traumatology. v. 22, p. 173-180. 2006.

38- VASCONCELLOS RRDH, et al. Trauma na dentição decídua: enfoque atual. Revista de Cirurgia e Traumatologia Buco-MaxiloFacial 2003; 3(2): 17-24.

39- VIEGAS CM, et al. Predisposing factors for traumatic dental injuries in Brazilian preschool children. European Journal of Pediatric Dentistry, vol. 11/2-2010.

40- WANDERLEY MT, et al. Traumatismos nos dentes decíduos: entendendo sua complexidade. Revista da Associação Paulista de Cirurgiões Dentistas, v. 68, n. 3, p. 194-200, 2014. 